\title{
Steerability detection of arbitrary 2-qubit state via machine learning
}

\author{
Changliang Ren ${ }^{1,2, *}$ and Changbo $\mathrm{Chen}^{3, *}$ \\ ${ }^{1}$ Center for Nanofabrication and System Integration, \\ Chongqing Institute of Green and Intelligent Technology, \\ Chinese Academy of Sciences, Chongqing 400714, People's Republic of China ${ }^{\dagger}$ \\ ${ }^{2}$ CAS Key Laboratory of Quantum Information, University of Science and Technology of China, Hefei 230026, PR China \\ ${ }^{3}$ Chongqing Key Laboratory of Automated Reasoning and Cognition, \\ Chongqing Institute of Green and Intelligent Technology, \\ Chinese Academy of Sciences, Chongqing 400714, People’s Republic of China ${ }^{\ddagger}$
}

(Dated: March 7, 2019)

\begin{abstract}
Quantum steering is an important nonclassical resource for quantum information processing. However, even lots of steering criteria exist, it is still very difficult to efficiently determine whether an arbitrary two-qubit state shared by Alice and Bob is steerable or not, because the optimal measurement directions of Alice are unknown. In this work, we provide an efficient quantum steering detection scheme for arbitrary 2-qubit states with the help of machine learning, where Alice and Bob only need to measure in a few fixed measurement directions. In order to prove the validity of this method, we firstly realize a high performance quantum steering classifier with the whole information. Furthermore, a high performance quantum steering classifier with partial information is realized, where Alice and Bob only need to measure in three fixed measurement directions. Our method outperforms the existing methods in generic cases in terms of both speed and accuracy, opening up the avenues to explore quantum steering via the machine learning approach.
\end{abstract}

PACS numbers:

\section{INTRODUCTION}

In the great debate of quantum mechanics in 1930s, Einstein, Podolsky, and Rosen (EPR) [1] challenged the completeness of quantum mechanics (QM) based on local realism usually called EPR paradox. It points out a way to deeply investigate the difference or conflict between classical theory and quantum theory. Especially, three types of quantum correlations originated from EPR paradox: quantum entanglement [2], Bell nonlocality [3], and EPR steering [4], have been put forward. Within the hierarchy of nonlocalities, the set of EPR steerable states is a subset of entangled states and a superset of Bell nonlocal states. Quantum entanglement and Bell nonlocality have attained flourishing developments since 1964. However, a rigorous formulation of the concept of EPR steering was not elaborately interpreted until 2007 [5]. Recently, it has gained increasing interest in quantum optics and quantum information communities [57]. For instance, EPR steering can provide security in one-sided device-independent quantum key distribution (1SDI-QKD) [8-10] and play an operative role in channel discrimination [11] and teleamplification [12].

Naturally, detection and characterization of steering have attracted increasing attention $[5-7,11,13-24]$. Various steering criteria and inequalities have been derived, such as linear steering inequalities [15, 18, 22], inequalities based on multiplicative variances [13-15], entropic uncertainty relations $[16,17]$, fine-grained uncertainty relations [19], and hierarchy of steering criteria based on moments [20]. In particular, a necessary and sufficient condition for a two-qubit state to be steerable with re- spect to projective measurements is exhibited [24]. Actually, these criteria can be computed through semidefinite programming [25].

For an arbitrary quantum state shared by Alice and Bob, to determine if Alice can steer Bob, those criteria boils down to finding optimal measurement directions of Alice, which is resource demanding as explained below. In real experimental test, if Alice and Bob share an unknown state, there are two ways to identify the steerability of this state. One is computing through the steering criteria after a complete quantum state tomograph measurement, the other is trying to directly observe the characterized phenomenon (such as the violation of the steering inequality), which can distinguish the steerability and non-steerability. Obviously, the former needs to measure the whole information of the state, while the latter has to try many times by choosing a large amount of measurement directions until the characterized phenomenon is observed. Hence, both of them need a lot of measurements and not efficient. It is even worse when there are a large amount of different states to be detected, which is typical if one wants to detect steerability of a sequence of distinct rapidly generated states. Thus it remains challenging to develop an efficient approach to detect steerability for experimental test.

Recently, the successful applications of machine learning approach on entanglement [26, 27] and nonlocality discriminants [28] shed a new light on this problem. Machine learning possesses the capability to instantly make predictions on new data with reasonable accuracy after learning from large amount of existing data. In the past few decades, there has been a rapid growing interest not 
only in theoretical studies, but also in a variety of applications of machine learning. Interestingly, beside its extensive applications in industry, machine learning has also been employed to investigate physics-related problems in recent years. Nowadays, many quantum implementations of machine learning have been introduced to achieve better performance for quantum information processing [26-37], such as the hamiltonian learning [34], automated quantum experiments search [35], phase transition identification [36], identification of topological phase of matter [37], entanglement classification [26-28], just to name a few.

Certainly, these works motivate us to adopt machine learning as an alternative approach for investigations of various quantum tasks. Different from the previous researches, in this paper, we employ the machine learning techniques to tackle the bipartite steering detection problem by recasting it as a learning task. We build several new steerability classifiers underpinned by machine learning techniques. Firstly, an efficient steerability classifiers with the whole information demonstrated the validity of steering classification by machine learning. Secondly, and more importantly, we provide a quantum steering detection scheme for arbitrary two-qubit states with the help of machine learning, where Alice and Bob only need to measure in three fixed measurement directions. These efficient steerability classifiers, which work for arbitrary 2-qubit states, are exhibited and fully analyzed. Either for arbitrary 2-qubit state or special states, they can perform better than the traditional semidefinite programming (SDP). More importantly, comparing with the classical method, this approach is much less resource demanding and can quickly determine whether a state is steerable with a well-trained classifier. Hence, it provides a simpler and more efficient way to detect steerability, which sheds new light on classification of quantum steering with limited resources, and represents a step towards large-scale machine-learning-based applications in quantum information processing.

\section{QUANTUM STEERING}

We start by defining the scenario in which quantum steering is discussed. For the sake of convenience, let us only consider the simplest case - two qubit system. Consider a bipartite situation composed by Alice and Bob sharing an arbitrary quantum state $\rho$. Suppose Alice performs measurement $\hat{A}$ with outcome $a$ and Bob performs measurement $\hat{B}$ with outcome $b$. These outcomes are thus in general governed by a joint probability distribution $P(a, b \mid \hat{A}, \hat{B}, \rho)$. Such joint probability distribution predicted by quantum theory is defined by

$$
P(a, b \mid \hat{A}, \hat{B}, \rho)=\operatorname{Tr}\left(\hat{M}_{A}^{a} \otimes \hat{M}_{B}^{b} \rho\right),
$$

where $\hat{M}_{A}^{a}$ and $\hat{M}_{B}^{b}$ are the projective operators for Alice and Bob respectively.

It is well-known that, Wiseman, Jones and Doherty formally defined quantum steering as the possibility of remotely generating ensembles that could not be produced by a local hidden state (LHS) model. The mathematic formulation of the LHS model adds an extra requirement on Bob's probabilities, which can be expressed as

$$
\begin{aligned}
P(a, b \mid \hat{A}, \hat{B}, \rho) & =\sum_{\lambda} P(\lambda) P(a \mid \hat{A}, \lambda) P_{Q}(b \mid \hat{B}, \lambda) \\
P_{Q}(b \mid \hat{B}, \lambda) & =\operatorname{Tr}\left(\rho_{\lambda} \hat{M}_{B}^{b}\right),
\end{aligned}
$$

where $\rho_{\lambda}$ is a qubit specified by $\lambda$. If the joint probability can be decomposed in the form of Eq. (2), then we say that Alice can not steer Bob's state. Otherwise $P(a, b \mid \hat{A}, \hat{B}, \rho)$ shows quantum steering correlation (in the sense that Alice steers Bob). The steering scenario consists of the situation where no characterisation of Alice's measurements is assumed, while Bob has full control of his measurements and can thus access the unnormalised conditional states $\sigma_{a \mid A}$, where $\sigma_{a \mid A}=$ $\operatorname{Tr}_{A}\left[\left(\hat{M}_{A}^{a} \otimes I\right) \rho\right]$. In other words, deciding whether an assemblage $\sigma_{a \mid A}$ demonstrates steering amounts to checking whether there exists a collection of quantum states $\rho_{\lambda}$ and probability distributions $P(\lambda)$ and $P(a \mid \hat{A}, \lambda)$ such that (2) holds. Obviously this is in principle a hard problem, since the variable $\lambda$ could assume infinitely many values. However, if the number of measurements and outputs is finite, this problem becomes much simpler, and it was shown in [4] that the problem can be solved through semi definite programming (SDP) [38]. Next, we briefly review this approach.

Suppose that Alice performs $m$ measurements, labeled as $x=0,1 \ldots \ldots, m-1$. One can write a SDP that determines if Alice can steer Bob [4],

$$
\begin{array}{ll}
\text { given } & \left\{\sigma_{a \mid x}\right\},\left\{D\left(a \mid x, \lambda^{\prime}\right)\right\}_{\lambda^{\prime}} \\
\min _{\left\{F_{a \mid x}\right\}} & \operatorname{Tr} \sum_{a x} F_{a \mid x} \sigma_{a \mid x} \\
\text { s.t. } & \sum_{a x} F_{a \mid x} D\left(a \mid x, \lambda^{\prime}\right) \geq 0 \quad \forall \lambda^{\prime} \\
& \operatorname{Tr} \sum_{a x, \lambda^{\prime}} F_{a \mid x} D\left(a \mid x, \lambda^{\prime}\right)=1,
\end{array}
$$

where $F_{a \mid x}$ are Hermitian matrices, $\lambda^{\prime}$ is a map from $\{x\}$ to $\{a\}$ and $D\left(a \mid x, \lambda^{\prime}\right)=\delta_{a, \lambda^{\prime}(x)}$, that is $D\left(a \mid x, \lambda^{\prime}\right)=1$ if $\lambda^{\prime}(x)=a$ and $D\left(a \mid x, \lambda^{\prime}\right)=0$ if $\lambda^{\prime}(x) \neq a$. If the objective value of (3) is negative for some measurements $x$, then $\rho$ is steerable from Alice to Bob. On the other hand, a non-negative value means that there exists an LHS model.

\section{QUANTUM STEERING CLASSIFIER WITH WHOLE INFORMATION}

Theoretically, we can detect steering more and more precise with the increase of measurements by SDP [25]. 
However, there is yet a noticeable drawback of the above SDP approach from the perspective of the tradeoff between the accuracy and time consumption. Boosting the accuracy means adding additional extreme points to enlarge the convex hull, which requires more time to determine if a point is inside the enlarged convex hull or not. To overcome this, we combined SDP with supervised learning, as machine learning has the power to speed up such computations.

Naturally, the bipartite steering detection problem can be formulated as a supervised binary classification task. Here, the datasets are generated by adopting the following procedure:

- First generating two random $4 \times 4$ matrices $M$ and $N$, which are used to generate a Hermitian matrix $H:=(M+i N)(M+i N)^{\dagger}$, where $\dagger$ means taking the conjugate transpose, and a density matrix $\rho_{A B}:=$ $H / \operatorname{Tr}(H)$.

- Since $\rho_{A B}$ is a density matrix of $4 \times 4$, it is enough to use the first 3 elements on the diagonal and the real and imaginary parts of 6 elements below the diagonal of the matrix to form the vector of features, which is a real vector of 15 numbers in the interval $(-1,1)$, denoted by $F_{1}$.

- For a given density matrix $\rho_{A B}$, we run SDP Program (3) 100 times with different values of measurements. If the objective value is negative, we assign a label -1 ; otherwise we assign a label +1 , which means that we do not know if Alice can steer Bob.

For each $m=2, \ldots, 8$, we generate the corresponding dataset until at least 5000 samples with label +1 and 5000 samples with label -1 are obtained. Generating the datasets for all different settings $m=2, \ldots, 8$ take several months on a workstation. Here, we should emphasize that the collected states for different settings $m$ are totally random and independent. Finally, we collected over 70000 samples in total [39]. Actually, it becomes harder and harder to obtain a dataset with the increase of amount of measurements. For example, when $m=8$, it spent about 63 days to collect the dataset. For each $m=2, \ldots, 8$, the last 1000 positive samples and the last 1000 negative samples are reserved for test. The rest 4000 positive samples and 4000 negative samples are kept as the training set to learn a classifier. We employ a 4 -fold cross-validation technique and a grid search approach for selecting best hyperparameters. The machine learning method we use is support vector machine (SVM).

SVM is a supervised learning model used for classification and regression analysis, which requires solving the

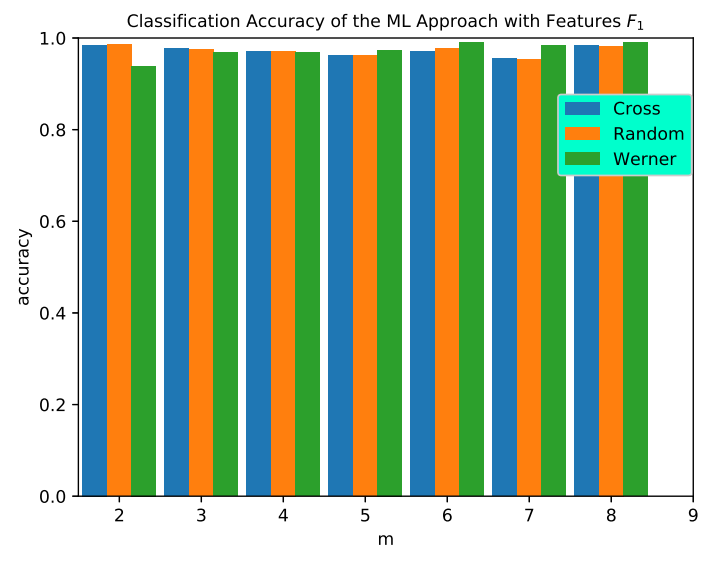

FIG. 1: (Color online) Classification accuracy of machine learning with the whole information ( $F_{1}$-features). The first column (blue) depicts the accuracy of cross validation; the second column (orange) depicts the classification accuracy on random states and the third column (green) depicts the classification accuracy on the Werner state.

following optimization problem:

$$
\begin{array}{ll}
\text { given } & \left(\mathbf{x}_{i}, y_{i}\right), i=1, \ldots, \ell \\
\min _{\mathbf{w}, b, \xi} & \frac{1}{2} \mathbf{w}^{T} \mathbf{w}+C \sum_{i=1}^{\ell} \xi_{i} \\
\text { s.t. } & y_{i}\left(\mathbf{w}^{T} \phi\left(\mathbf{x}_{i}\right)+b\right) \geq 1-\xi_{i} \\
& \xi_{i} \geq 0 .
\end{array}
$$

Here $\ell$ is the number of samples, $y_{i}$ and $\mathbf{x}_{i}$ are respectively the label and the vector of features of sample $i$, $\phi$ is a mapping implictly defined by a kernel function and we choose the radial basis function (RBF) kernel $K\left(\phi\left(\mathbf{x}_{i}\right)^{T}, \phi\left(\mathbf{x}_{j}\right)\right)=\exp \left(-\gamma\left\|\mathbf{x}_{i}-\mathbf{x}_{j}\right\|^{2}\right)$ with the parameters $C$ and $\gamma$ to be determined by a grid search approach when training the model.

In the rest of this section, the models are trained with feature vectors of type $F_{1}$, which encodes the full information of a two-qubit quantum state. After the SVM model is trained, we test the performance by creating a new set of quantum ensemble that is distinct from the data set employed for training. The classification accuracy of the learning model for each $m$ is illustrated in Fig. 1. All the accuracies for training and cross validation are higher than 0.95 , which clearly shows that the models are well-trained.

It is reasonable to predict that, if these steerability classifiers are well-trained, the classifiers should turn more precise with the increase of $m$. To show such validity, we use classifiers learned for different $m$ to test against the random data for $m=8$. As illustrated in Fig. 2, the blue-circle line is for $F_{1}$ features, it is shown that, the error drops very rapidly (except $m=6$, which may come from the imperfection in the learning pro- 


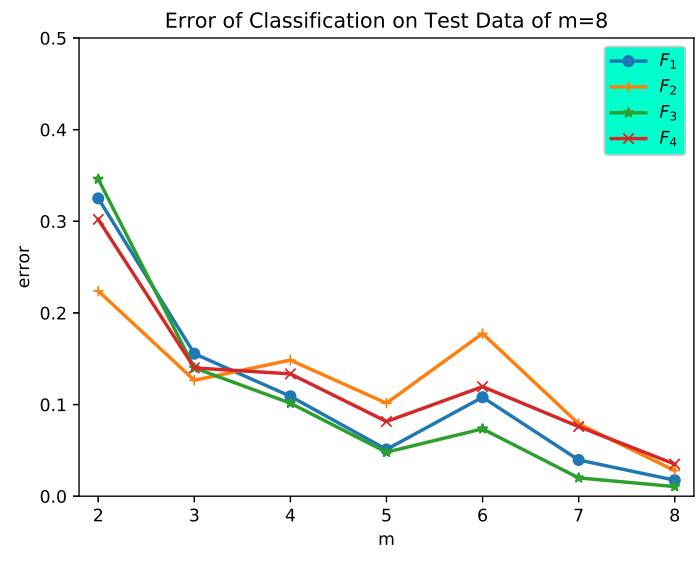

FIG. 2: (Color online) Error of classification on test data of $m=8$ by each machine learning classifier with different features $F_{1}, F_{2}, F_{3}, F_{4}$ (summarized in appendix) respectively.

cess). However, the variation tendency is identical to the theoretical prediction in general (the more measurement settings, the more precise the prediction) which further demonstrates that these are well-trained classifiers of quantum steering.

To demonstrate the generalization ability of the steering classifiers, we study their ability in clarifying a special state which has unambiguous bound for steerability. The state can be written as,

$$
\rho_{\mathrm{W}}=p|\psi\rangle\langle\psi|+(1-p) \rho_{\mathrm{A}} \otimes I / 2
$$

where $|\psi\rangle=\cos \xi|00\rangle+\sin \xi|11\rangle, \rho_{\mathrm{A}}=\operatorname{Tr}_{\mathrm{B}}(|\psi\rangle\langle\psi|)$. This state is a two-qubit one-way steerable state, which was exhibited by Bowles et.al in [40] recently. The state reduces to the Werner state when $\xi=\frac{\pi}{4}$. In simplicity, this state can be called "generalized Werner state". Different from entanglement and nonlocality, each qubit in Alice and Bob plays different role in the steering scenario. There exists one-way quantum steering. That is, special entangled states such that steering can occur from Alice to Bob, but not from Bob to Alice. One-way steering states attracted more attention due to their special characterization. For the state in Eq. (5), which is unsteerable from Alice to Bob [40], if

$$
\cos ^{2} 2 \xi \geq \frac{2 p-1}{(2-p) p^{3}}
$$

which has also been experimentally demonstrated very recently in [41]. Obviously, the bound of the parameter $p$ that Alice can steer Bob's state is determined by Eq. (6). Here we apply our classifiers to predict the steerability of such states. The test states are constructed according to the uniform distribution of $p$ and $\xi$. For each $\xi=\left\{\frac{\pi}{4}, \frac{\pi}{6}, \frac{\pi}{8}, \frac{\pi}{12}\right\}$, we generate 10000 test samples.
We predict the steerability bounds using both learned classifiers and SDP.
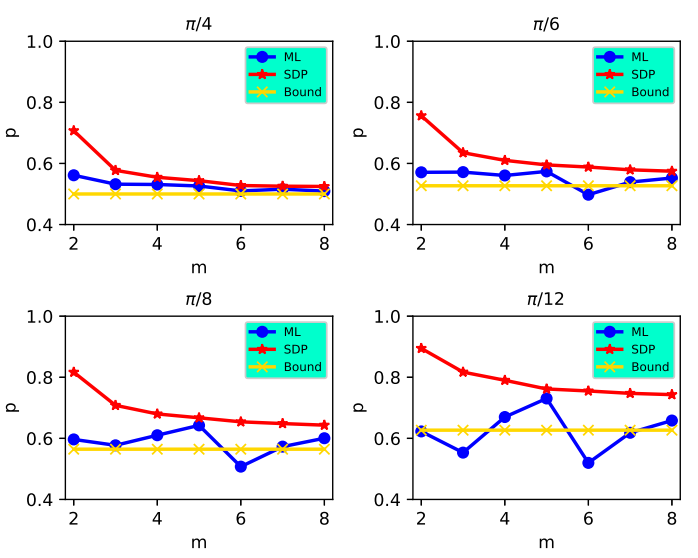

FIG. 3: (Color online) The predictions of steerability for generalized Werner states by learned classifiers and SDP. The blue line is the result predicted by learning classifiers with $F_{1}$ features. Similarly, the red line is the result predicted by SDP. And the orange line is the steerability bound from Alice to Bob.

As illustrated in Fig. 3, four subfigs in this picture correspond to $\xi=\left\{\frac{\pi}{4}, \frac{\pi}{6}, \frac{\pi}{8}, \frac{\pi}{12}\right\}$ respectively. In each subfig, the blue line is the result predicted by the learned classifiers for $m=2, \ldots, 8$ respectively. Similarly, the red line is the result predicted by SDP with $m=2, \ldots, 8$. The orange line is the steerability bound from Alice to Bob which is defined by Eq. (6). Obviously, the learning classifiers perform better than the traditional SDP. Especially, when $\xi=\frac{\pi}{4}$, Werner state, the learning classifiers demonstrate the best performance. In Fig. 1, the third green column depicts the classification accuracy on the Werner state. It is interesting to notice that for $m>4$, the classification accuracy on Werner state is even higher than on random data, despite the fact that the model is trained with random data. As the decrease of $\xi$, the prediction errors of both the learning classifiers and SDP increase. It is a reasonable phenomenon since the predictions for the marginal states become harder and harder. In Fig. 4 , the first subfig for $F_{1}$ features shows the classification error for generalized Werner States (with different angles). Obviously, the error increases when the angle drops which coincides with the above analysis. Another interesting phenomenon is, even though the learned classifiers can be more effective than SDP, it is still possible that they may predict the value of $p$ lower than the steerability bound, which almost never happens for SDP. The reason is that, one typical character for machine learning classifiers is that they can predict the positive to be negative and vice versa. However, the main error for SDP occurs when it predicts the negative to be positive. Hence, this phenomenon can be used to distinguish which 
method is used.
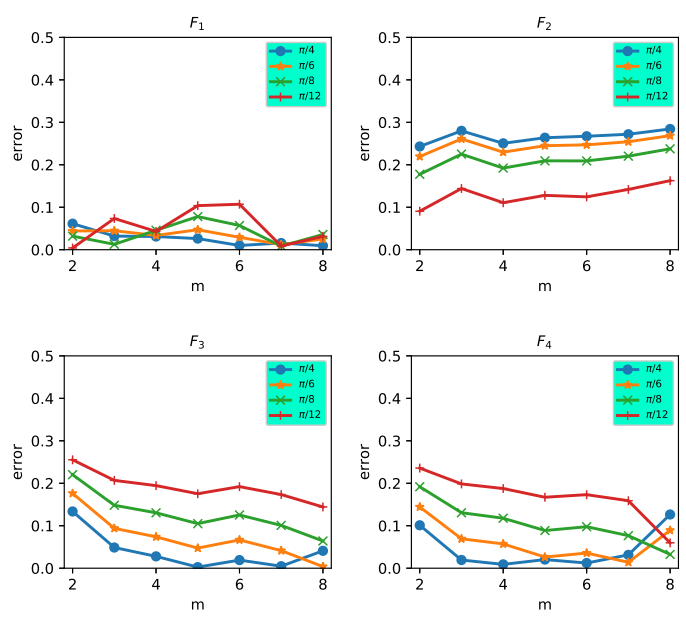

FIG. 4: (Color online) Classification error for generalized Werner states with different classifiers.

The above results clearly demonstrate the validity of steerability detection by machine learning. Even the whole information is still needed by this scheme, same as the traditional SDP method, the machine learning method is much more efficient than SDP in data processing. Take $m=8$ for example, the learned classifier spends about $10^{-2} \mathrm{~s}$ to predict an unknown state while it takes about $10^{2} \mathrm{~s}$ for the SDP with $m=8$. Maybe it is unfair to exhibit the time advantage in testing only one state, after all the time cost of the machine learning classifier should contain both the training time and the prediction time. However, when the task is to predict a large number of unknown states, the time advantage of machine learning classifier is obvious.

\section{QUANTUM STEERING CLASSIFIERS WITH PARTIAL INFORMATION}

Although the above steering classifier via machine learning boosts the performance of the state classification compared with traditional SDP method, it still has a disadvantage that such classifier needs the whole information of the state as the input feature. However, the size of a quantum state grows exponentially when scaled up, which makes large-scale quantum state tomography intractable to carry out. Hence, it will become more and more difficult to extend the method to higher dimensions. Hence, it is important to further explore the possibility of learning with only partial information of the quantum state. Here we will introduce an efficient quantum steering detection scheme for arbitrary two-qubit states with the help of machine learning, where Alice and Bob only

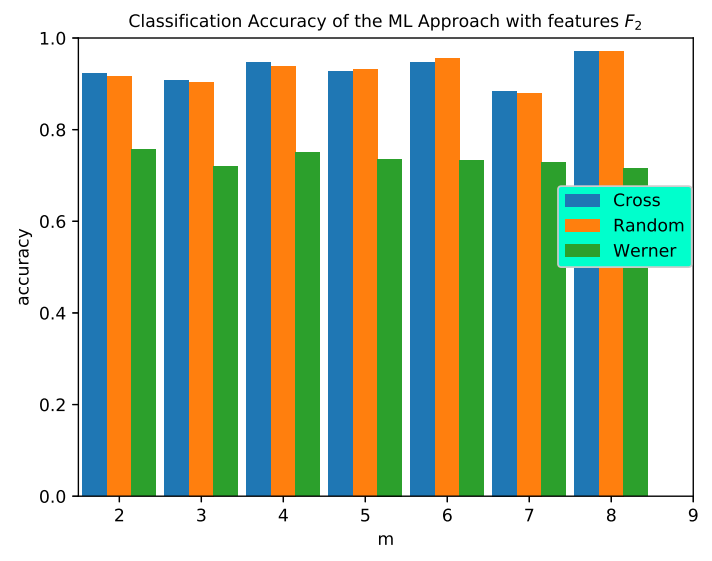

FIG. 5: (Color online) Classification accuracy of machine learning with partial information ( $F_{2}$ features). The first column (blue) depicts the accuracy of cross validation; the second column (orange) depicts the classification accuracy on random states and the third column (green) depicts the classification accuracy on the Werner state.

need to measure in a few fixed measurement directions.

Steerability is unaffected by local unitaries for Alice and "local filters" /"stochastic local operations" for Bob. Hence the relevant information for steerability could be encoded in a smaller feature vector. Actually, an arbitrary two-qubit state can be expressed in the local Pauli basis

$\rho=\frac{1}{4}\left(I+\sum_{i=1}^{3} r_{i} \sigma_{i} \otimes I+\sum_{j=1}^{3} s_{j} I \otimes \sigma_{j}+\sum_{k, l=1}^{3} \tau_{k l} \sigma_{k} \otimes \sigma_{l}\right) .(7)$

Steerability is determined by all the parameters $r_{i}, s_{j}, \tau_{k l}$. It is intuitively believed that steerability is dominated by the correlation terms $\tau_{k l}$ between the two qubits from Eq. (7). Hence, it is natural to extract the coefficients of the correlation terms, $\left\{\tau_{k l}\right\}$, as features. More precisely, the partial information is extracted by computing $\operatorname{Tr}\left[\left(\sigma_{k} \otimes \sigma_{l}\right) \rho\right]$ as features, denoted by $F_{2}$.

We repeat the same training and test process as for $F_{1}$. The classification accuracy of the learned models for each $m$ is illustrated in Fig. 5. It is interesting to see that, even the classification accuracy on random quantum state is apparently high, the accuracy for classifying Werner states is rather low. Similarly, the second subfigure of Fig. 4 shows that the classification errors for generalized Werner states are high. Thus the models trained with features $F_{2}$ have poor generalization ability. As a result, these classifiers have poor performance compared with traditional SDP method, as illustrated by Fig. 6. Therefore, exploring high performance classifier with partial information is not trivial, and such a simple and crude way is impracticable.

To further explore high performance classifier with partial information, we convert the state $\rho$ into a canonical 

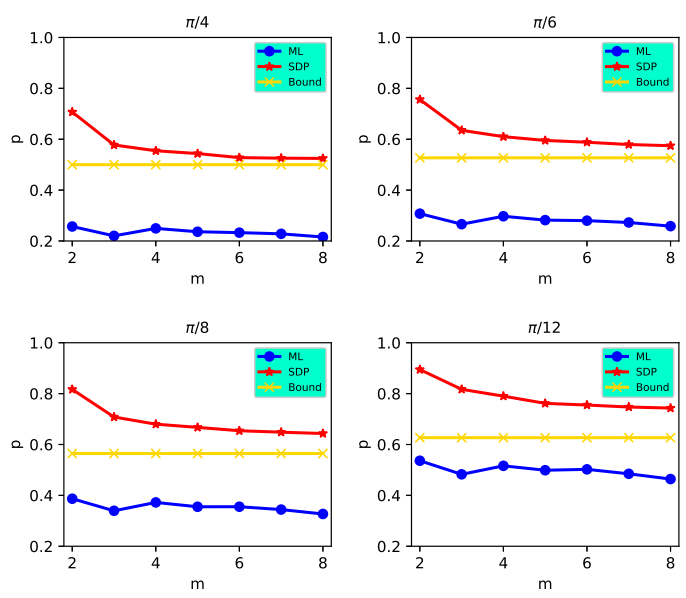

FIG. 6: (Color online) The predictions of steerability for generalized Werner states by learning classifiers and SDP. The blue line is the result which predicted by learning classifiers with $F_{2}$ features. Similarly, the red line is the result which predicted by SDP. And the orange line is the steerability bound from Alice to Bob.

form $\rho_{0}$ by local unitaries, which preserves the steerability of $\rho$. As proved in [40], the map given by,

$$
\rho_{0}=\left(\mathrm{I} \otimes \rho_{B}^{1 / 2}\right) \rho\left(\mathrm{I} \otimes \rho_{B}^{1 / 2}\right)
$$

where $\rho_{B}=\operatorname{Tr}_{A}[\rho]$, preserves the steerability of $\rho$. The interesting property of this map is that when applied to an arbitrary state $\rho$, it can be realized by only local operation on Bob.

Similarly, we can extract the coefficients of the correlation terms of the resulting state $\rho_{0}, \tau_{k l}$, to combine a real vector of 9 numbers as features. More precisely, we compute $\operatorname{Tr}\left[\left(\sigma_{k} \otimes \sigma_{l}\right) \rho_{0}\right]$ as features, denoted by $F_{3}$. The classification accuracy of learned models for each $m$ is illustrated in Fig. 7. In general, all the accuracies turn to higher and higher $(>0.95)$ with the increase of $m$, which clearly shows that we get several well-trained learning machines.

Moreover, we observe the following similar phenomenon as using the full information features. As illustrated in Fig. 2, the green-star line is for $F_{3}$ features, it is shown that, the error drops very rapidly. Hence, the variation tendency is identical to the theoretical prediction in general (the more measurement settings, the more precise the prediction).

Fig. 8 illustrates the steerability bounds predicted by machine learning classifiers and SDP with the angle $\xi=\left\{\frac{\pi}{4}, \frac{\pi}{6}, \frac{\pi}{8}, \frac{\pi}{12}\right\}$ respectively. Obviously, the learned classifiers outperforms the traditional SDP except for the states when $\xi=\frac{\pi}{12}$, which is near the boundary of steerability. As the decrease of $\xi$, the prediction er-

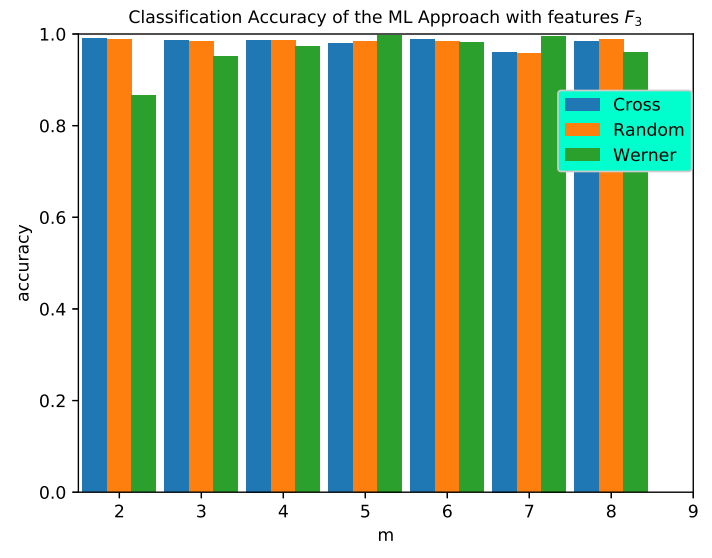

FIG. 7: (Color online) Classification accuracy of machine learning with partial information ( $F_{3}$ features). The first column (blue) depicts the accuracy of cross validation; the second column (orange) depicts the classification accuracy on random states and the third column (green) depicts the classification accuracy on the Werner state.

rors of both the learning classifiers and SDP increase. As we mentioned for features $F_{1}$, it is a reasonable phenomenon since the predictions for the marginal states become harder and harder. In Fig. 4, the third subfig shows the classification error for generalized Werner States (with different angles) for $F_{3}$ features. Obviously, the error increases when the angle drops which coincides with the above analysis. Generally, this classifier has better performance compared with traditional SDP method.

Note that in this scheme, for any unknown state, Alice and Bob only need to measure in three fixed measurement directions. Therefore, it is more efficient than SDP in both physical measurement process and data processing. In particular, it should be very efficient for testing a large amount of arbitrary states in quantum information process, such as one-sided device-independent quantum key distribution (1SDI-QKD), channel discrimination and teleamplification, etc.

To explore the performance of machine learning based quantum steering using even less information, according to the symmetry, we dropped the coefficients of the correlation terms, $\sigma_{y} \otimes \sigma_{x}, \sigma_{z} \otimes \sigma_{x}, \sigma_{z} \otimes \sigma_{y}$ from $F_{3}$ and named the rest features as $F_{4}$. The training process was carried out as before. As illustrated in Fig. 9, the classification accuracy of such classifiers on random states is acceptable but lower than with $F_{3}$ features. Interestingly, it performs better on Werner states as illustrated in Fig. 10 except for $m=8$. The fact that the accuracy drops for $m=8$ may be caused by overfitting, as shown in Fig. 9. Similarly, the predictions for the marginal states becomes harder and harder. Even such classifiers with $F_{4}$ features perform worse than those with $F_{3}$ features, they are still more effective than those with $F_{2}$ features. 

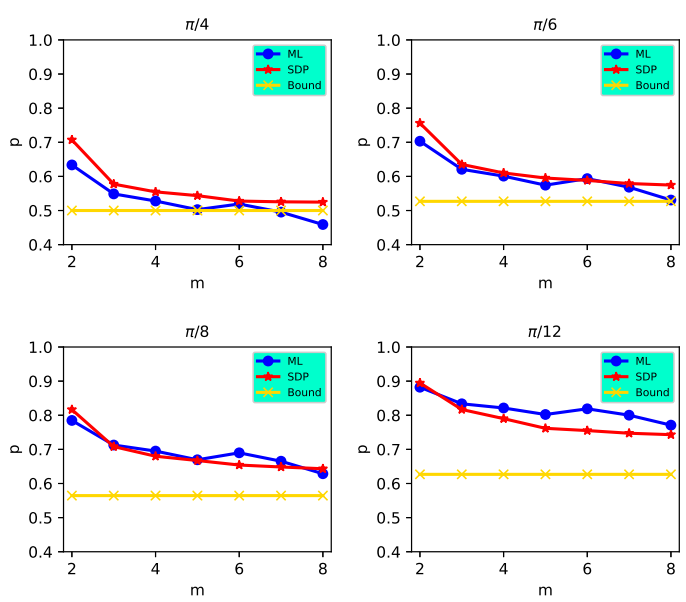

FIG. 8: (Color online) The predictions of steerability for generalized Werner states by machine learning classifiers and SDP. The blue line is the result predicted by learned classifiers with $F_{3}$ features. Similarly, the red line is the result which predicted by SDP. And the orange line is the steerability bound from Alice to Bob.

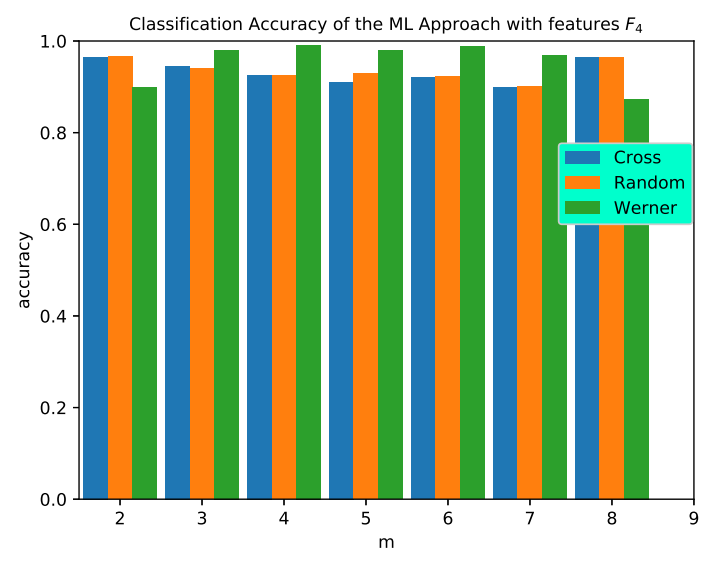

FIG. 9: (Color online) Classification accuracy of machine learning with partial information (F4 features). The first column (blue) depicts the accuracy of cross validation; the second column (orange) depicts the classification accuracy on random states and the third column (green) depicts the classification accuracy on the Werner state.

Hence, correctly extracting partial information is very important for realizing high performance steering classifiers via machine learning.

\section{CONCLUSION}

In this work, we have applied a method of machine learning to solve problems of quantum state classifica-
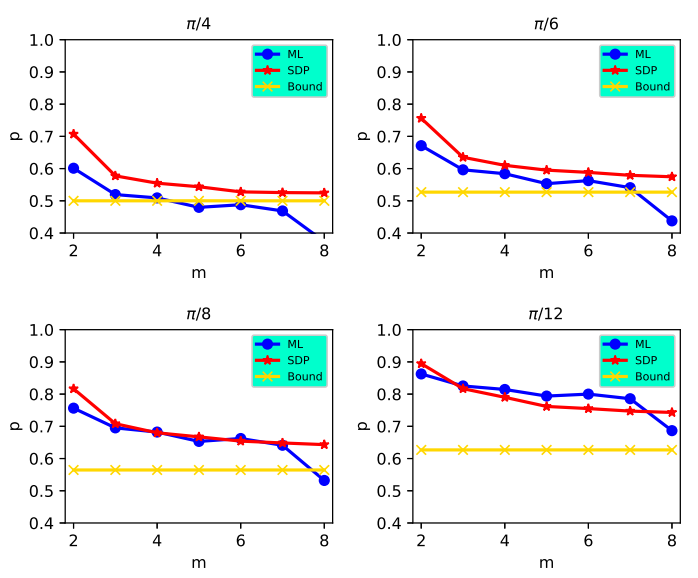

FIG. 10: (Color online) The predictions of steerability for generalized Werner states by learning classifiers and SDP. The blue line is the result predicted by learning classifiers with $F_{4}$ features. Similarly, the red line is the result predicted by SDP. And the orange line is the steerability bound from Alice to Bob.

tion in quantum information science. Several reliable enhanced steerability classifiers by combining supervised learning and the SDP method are achieved.

At first, we build a high performance quantum steering classifier with the whole information, which are used to test some random unknown states and the generalized Werner states. The prediction performance of such learning classifier and SDP are completely analyzed and discussed. It clearly demonstrates the validity and efficiency of steering classification by machine learning. Secondly, we investigate the possibility of constructing steering classifiers with partial information. It is shown that, correctly extracting partial information is very important for realizing high quality steering classifiers via machine learning. Finally, an efficient quantum steering detection scheme for arbitrary two-qubit states via machine learning is realized, where Alice and Bob only need to measure in three fixed measurement directions. It should be very efficient for testing the steerability of a large amount of arbitrary states in quantum information process, such as one-sided device-independent quantum key distribution (1SDI-QKD), channel discrimination and teleamplification, etc.

Acknowledgement.-C.L.R. is supported by National key research and development program (No. 2017YFA0305200), the Youth Innovation Promotion Association (CAS) (No. 2015317), the National Natural Science Foundation of China (No. 11605205), the Natural Science Foundation of Chongqing (No. cstc2015jcyjA00021, cstc2018jcyjAX0656), the Entrepreneurship and Innovation Support Program for Chongqing Overseas Returnees (No.cx017134), the fund 
of CAS Key Laboratory of Microscale Magnetic Resonance, and the fund of CAS Key Laboratory of Quantum Information. And C.C. is supported by the National Natural Science Foundation of China (No. 11771421, 11471307, 61572024, 11671377), cstc2018jcyj-yszxX0002 of Chongqing, and the Key Research Program of Frontier Sciences of CAS (QYZDB-SSW-SYS026). C. L. Ren and C. B. Chen contributed equally to this work.

* These authors contributed equally.

† Electronic address: renchangliang@cigit.ac.cn

‡ Electronic address: chenchangbo@cigit.ac.cn

[1] A. Einstein, B. Podolsky, and N. Rosen, Phys. Rev. 47, 777 (1935).

[2] R. Horodecki, P. Horodecki, M. Horodecki, K. Horodecki, Rev. Mod. Phys. 81, 865 (2009).

[3] N. Brunner, D. Cavalcanti, S. Pironio, V. Scarani, and S.Wehner, Rev. Mod. Phys. 86, 419 (2014).

[4] D. Cavalcanti and P. Skrzypczyk, Rep. Prog. Phys. 80, 024001 (2017).

[5] H. M. Wiseman, S. J. Jones and A. C. Doherty, Phys. Rev. Lett. 98, 140402 (2007).

[6] S. J. Jones, H. M. Wiseman, and A. C. Doherty, Phys. Rev. A 76, 052116 (2007).

[7] P. Skrzypczyk, M. Navascus, and D. Cavalcanti, Phys. Rev. Lett. 112, 180404 (2014).

[8] C. Branciard, E. G. Cavalcanti, S. P. Walborn, V. Scarani, and H. M. Wiseman, Phys. Rev. A 85, 010301 (2012).

[9] T. Gehring et al., Nat. Commun. 6, 8795 (2015).

[10] N. Walk et al., Optica 3, 634 (2015).

[11] M. Piani and J. Watrous, Phys. Rev. Lett. 114, 060404 (2015).

[12] Q. He, L. Rosales-Zárate, G. Adesso, and M. D. Reid, Phys. Rev. Lett. 115, 180502 (2015).

[13] M. D. Reid, Phys. Rev. A 40, 913 (1989).

[14] M. D. Reid, P. D. Drummond,W. P. Bowen, E. G. Cavalcanti, P. K. Lam, H. A. Bachor,U. L.Andersen, and G. Leuchs, Rev. Mod. Phys. 81, 1727 (2009).

[15] E. G. Cavalcanti, S. J. Jones, H. M. Wiseman, and M. D. Reid, Phys. Rev. A 80, 032112 (2009).

[16] S. P. Walborn, A. Salles, R. M. Gomes, F. Toscano, and P. H. Souto Ribeiro, Phys. Rev. Lett. 106, 130402 (2011).

[17] J. Schneeloch, C. J.Broadbent, S. P.Walborn,E. G.Cavalcanti, and J. C. Howell, Phys. Rev. A 87, 062103 (2013).

[18] M. F. Pusey, Phys. Rev. A 88, 032313 (2013).

[19] T. Pramanik, M. Kaplan, and A. S. Majumdar, Phys. Rev. A 90, 050305 (2014).

[20] I. Kogias, P. Skrzypczyk, D. Cavalcanti, A. Acn, and G. Adesso, Phys. Rev. Lett. 115, 210401 (2015).

[21] I. Kogias, A. R. Lee, S. Ragy, and G. Adesso, Phys. Rev.
Lett. 114, 060403 (2015).

[22] E. G. Cavalcanti, C. J. Foster, M. Fuwa, H. M. Wiseman, J. Opt. Soc. Am. B, 32, No. 4, A74 (2015).

[23] H. Zhu, M. Hayashi, L. Chen, Phys. Rev. Lett. 116, 070403(2016).

[24] H. C. Nguyen, T. Vu, Europhys. Lett. 115, 1 (2016).

[25] D. Cavalcanti, and P. Skrzypczyk, Rep. Prog. Phys. 80, 2 (2016).

[26] Y. C. Ma and M. H. Yung, arXiv: 1705.00813.

[27] S. R. Lu et.al, arXiv: 1705.01523.

[28] D. L. Deng, X. Li and S. D. Sarma, Phys. Rev. X 7, 021021 (2017).

[29] A. Assion, T. Baumert, M. Bergt, T. Brixner, B. Kiefer, V. Seyfried, M. Strehle and G. Gerber, Science 282, 919 (1998).

[30] M. Sasaki and A. Carlini, Phys. Rev. A 66, 022303 (2002).

[31] A. Bisio, G. Chiribella, G. M. DAriano, S. Facchini and P. Perinotti, Phys. Rev. A 81, 032324 (2010).

[32] A. Hentschel and B. C. Sanders, Phys. Rev. Lett. 104, 063603 (2010).

[33] J. Bang, S. W. Lee, H. Jeong and J. Lee, Phys. Rev. A 86, 062317 (2012).

[34] N. Wiebe, C. Granade, C. Ferrie, and D. G. Cory, Phys. Rev. Lett. 112, 190501 (2014).

[35] M. Krenn, M. Malik, R. Fickler, R. Lapkiewicz, and A. Zeilinger, Phys. Rev. Lett. 116, 090405 (2016).

[36] S. S. Schoenholz, E. D. Cubuk, D. M. Sussman, E. Kaxiras, and A. J. Liu, Nat. Phys. 12, 469 (2016).

[37] Y. Zhang and E.-A. Kim, Phys. Rev. Lett. 118, 216401 (2017).

[38] L. Vandenberghe and S. Boyd, SIAM Review 38, 49 (1996).

[39] We have shared all of the detail of the programs and the whole data in http://www . arcnl .org/cchen/data/quantum.

[40] J. Bowles, F. Hirsch, M. T. Quintino, and N. Brunner, Phys. Rev. A 93, 022121 (2016).

[41] Y. Xiao, X-J. Ye, K. Sun, J-S. Xu, C-F. Li, and G-C. Guo, Phys. Rev. Lett. 118, 140404 (2017).

\section{APPENDIX: THE VECTOR OF FEATURES}

For an arbitrary quantum state $\rho$, the four different features used in this work is summarized as below:

\begin{tabular}{|c|c|}
\hline \hline $\mathrm{F}_{1}$ & $\rho_{i i}, i \in\{1,2,3\}$, the real and imaginary part of $\rho_{i j}, j>i$ \\
\hline $\mathrm{F}_{2}$ & $\operatorname{Tr}\left[\left(\sigma_{k} \otimes \sigma_{l}\right) \rho\right],\{k, l\} \in\{x, y, z\}$ \\
\hline $\mathrm{F}_{3}$ & $\rho \rightarrow \rho_{0}, \operatorname{Tr}\left[\left(\sigma_{k} \otimes \sigma_{l}\right) \rho_{0}\right],\{k, l\} \in\{x, y, z\}$ \\
\hline $\mathrm{F}_{4}$ & $\mathrm{~F}_{3}$ except for the terms of $\left\{\sigma_{y} \otimes \sigma_{x}, \sigma_{z} \otimes \sigma_{x}, \sigma_{z} \otimes \sigma_{y}\right\}$ \\
\hline \hline
\end{tabular}

